\title{
The Allen Telescope Array
}

David R. DeBoer, William J. Welch, John Dreher, Jill Tarter, Leo Blitz, et al.

David R. DeBoer, William J. Welch, John Dreher, Jill Tarter, Leo Blitz, Michael Davis, Matt Fleming, Douglas Bock, Geoffrey Bower, John Lugten, G. Girmay-Keleta, Larry R. D'Addario, Gerry R. Harp, Rob Ackermann, Sander Weinreb, Greg Engargiola, Doug Thornton, Niklas Wadefalk, "The Allen Telescope Array," Proc. SPIE 5489, Ground-based Telescopes, (28 September 2004); doi: 10.1117/12.551737

Event: SPIE Astronomical Telescopes + Instrumentation, 2004, Glasgow, United Kingdom 


\title{
The Allen Telescope Array
}

\author{
David DeBoer*a, William J. Welch ${ }^{\mathrm{b}}$, John Dreher ${ }^{\mathrm{a}}$, Jill Tarter ${ }^{\mathrm{a}}$, Leo, Blitz ${ }^{\mathrm{b}}$, Michael Davis ${ }^{\mathrm{a}}$, \\ Matt Fleming ${ }^{\mathrm{b}}$, Douglas Bock ${ }^{\mathrm{b}}$, Geoffrey Bower ${ }^{\mathrm{b}}$, John Lugten ${ }^{\mathrm{b}}$, Girmay-Keleta ${ }^{\mathrm{a}}$, Larry \\ D'Addario $^{a}$, Gerry Harpa ${ }^{\mathrm{a}}$, Rob Ackermann ${ }^{\mathrm{a}}$, Sander Weinreb ${ }^{\mathrm{c}}$, Greg Engargiola ${ }^{\mathrm{b}}$, Doug \\ Thornton $^{\mathrm{b}}$, Niklas, Wadefalk ${ }^{\mathrm{c}}$

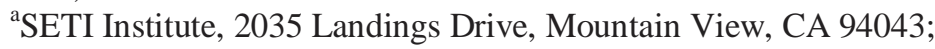 \\ ${ }^{\mathrm{b}}$ Radio Astronomy Laboratory, University of California, Berkeley, CA 94720 \\ ${ }^{\mathrm{c} J P L / C a l t e c h, ~} 4800$ Oak Grove Road, Pasadena, CA 91109
}

\begin{abstract}
The Allen Telescope Array, originally called the One Hectare Telescope (1hT) [1] will be a large array radio telescope whose novel characteristics will be a wide field of view (3.5 deg-GHz HPBW), continuous frequency coverage of 0.5 $11 \mathrm{GHz}$, four dual-linear polarization output bands of $100 \mathrm{MHz}$ each, four beams in each band, two $100 \mathrm{MHz}$ spectral correlators for two of the bands, and hardware for RFI mitigation built in. Its scientific motivation is for deep SETI searches and, at the same time, a variety of other radio astronomy projects, including transient (e.g. pulsar) studies, HI mapping of the Milky Way and nearby galaxies, Zeeman studies of the galactic magnetic field in a number of transitions, mapping of long chain molecules in molecular clouds, mapping of the decrement in the cosmic background radiation toward galaxy clusters, and observation of $\mathrm{HI}$ absorption toward quasars at redshifts up to $\mathrm{z}=2$. The array is planned for 350 6.1-meter dishes giving a physical collecting area of about 10,000 square meters. The large number of components reduces the price with economies of scale. The front end receiver is a single cryogenically cooled MIMIC Low Noise Amplifier covering the whole band. The feed is a wide-band log periodic feed of novel design, and the reflector system is an offset Gregorian for minimum sidelobes and spillover. All preliminary and critical design reviews have been completed. Three complete antennas with feeds and receivers are under test, and an array of 33 antennas is under construction at the Hat Creek Radio Observatory for the end of 2004. The present plan is to have a total of about 200 antennas completed by the summer of 2006 and the balance of the array finished before the end of the decade.
\end{abstract}

Keywords: Radio astronomy, antenna arrays

\section{Introduction}

Most science applications in radio astronomy require high sensitivity, and thus large amounts of collecting area and quality receivers. Building increasingly larger single dishes becomes difficult if not impossible, but is also generally undesirable for other reasons. One reason is that the resolution of a single dish is set by the diameter, and, in terms of the number of wavelengths, is generally small. For example, the world's largest single aperture, the telescope at the Arecibo Observatory in Puerto Rico (roughly $300 \mathrm{~m}$ in diameter) is about 1000 wavelengths in diameter at $1420 \mathrm{MHz}$, while a 6" optical telescope is about 280,000 wavelengths across in the visible. Increasing the resolution available at a given sensitivity is essential to make strong contributions in many areas of astrophysics. This decoupling of the resolution and sensitivity is done by splitting the aperture into many antennas, where now one can choose the sensitivity (number of antennas) independently of the resolution (spacing of the antennas).

Single dishes are also relatively inefficient at imaging as well. Incorporating focal plane arrays into the antenna receivers improves this a great deal (for some surveys to unmatched efficiency), however the size of the focal plane array is somewhat limited (such receivers are typically made with tens of detectors, not hundreds or thousands) and the resolution remains limited by the aperture diameter. Simplistically speaking, more degrees of freedom are better and single dishes typically have a limited number of degrees of freedom.

Lastly, large antenna structures are costly to build: Moore's law, with its seemingly inevitable improvement in performance and cost, does not govern steel and concrete costs. The economics of radio telescope building to a fixed sensitivity is a trade-off between antenna costs and receiver costs. The cost of a dish of a given diameter is a strong function of diameter (canonically a power of 2.7) and the cost of the electronics is a function of total number (more

*ddeboer@seti.org; phone 15106432329 
antennas often means having poorer receivers). Economics is the major driver, and, for best economy, the antenna and receiver costs should be comparable. For example, the VLA was built with 25-meter antennas because receiver costs were large when it was designed. With the advent of inexpensive electronics driven by cell phones, computers and the internet, receiver costs are dramatically lower, arguing for smaller antennas. The Allen Telescope Array (ATA) is the first telescope to be designed with this in mind, and includes other innovations to enhance the performance per cost ratio.

The Allen Telescope Array [1] is a joint project of the SETI Institute (Search for ExtraTerrestrial Intelligence, Mountain View, CA) and the Radio Astronomy Lab at the University of California, Berkeley. Conceived in a series of meetings convened by the SETI Institute in 1997-1999, the instrument was originally called the One Hectare Telescope (1hT), after its $10^{4}$ square meters of collecting area. Thanks to the generosity of the Paul G. Allen Foundations (Seattle, WA), the telescope has been funded through the research and development and initial construction phases. The Allen Telescope Array is currently under construction, with the first three antennas in place and a schedule that calls for 33 antennas by Fall 2004, 206 antennas by the summer of 2006, and 350 antennas sometime later in the decade. Figure 1 shows a rendering of how the 350 dishes will look at the Hat Creek Radio Observatory.

The scientific motivation for the facility is for deep SETI searches and, at the same time, a variety of other radio astronomy projects, including transient (e.g. pulsar) studies, HI mapping of the Milky Way and nearby galaxies, Zeeman studies of the galactic magnetic field in a number of transitions, mapping of long chain molecules in molecular clouds, mapping of the decrement in the cosmic background radiation toward galaxy clusters, and observation of $\mathrm{HI}$ absorption toward quasars at redshifts up to $\mathrm{z}=2$.

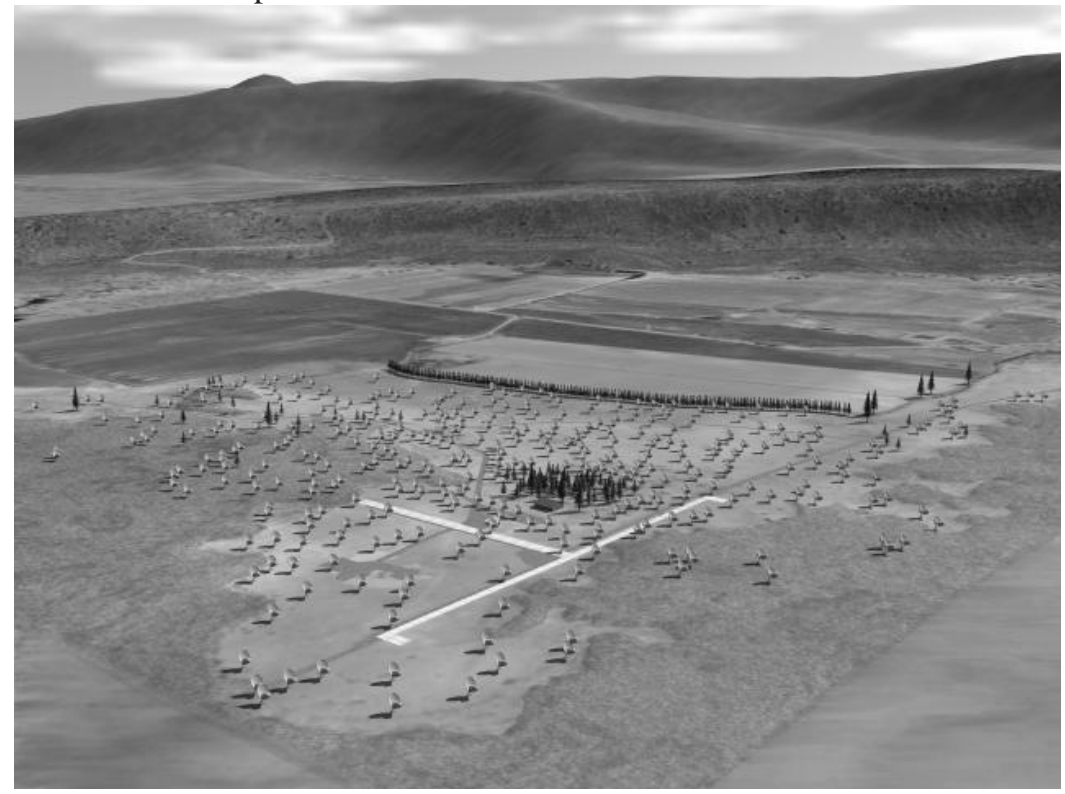

Figure 1: Rendering of the completed ATA-350 at the Hat Creek Radio Observatory (Credit Isaac Gary).

The technologies primarily enabling the ATA are low-cost ultra-wide-bandwidth analog electronics and high-speed digital electronics, as well as "mid-quantity" production techniques. This project has pushed the state of the art in many arenas such as feed design, antenna fabrication, photonic signal transport, and cryogenics. The primary driver has been to achieve high sensitivity and simultaneous wideband performance at a low cost. Technologies developed specifically by and for this project are ultra-wideband feeds, low-noise amplifiers, and low-cost analog fiber-optic links - ultrawideband here means spans of 22:1.

\section{Description}

The signal path for the Allen Telescope Array is shown in Figure 2 (see also Appendix A). Given the large field-ofview and the fact that the entire band from 0.5 to $11 \mathrm{GHz}$ is brought back to the lab, the ATA has a great deal of 
flexibility and concurrent-use potential. In fact, one central precept is that users from the SETI Institute and from the Radio Astronomy Lab be able to effectively and independently conduct concurrent observations.

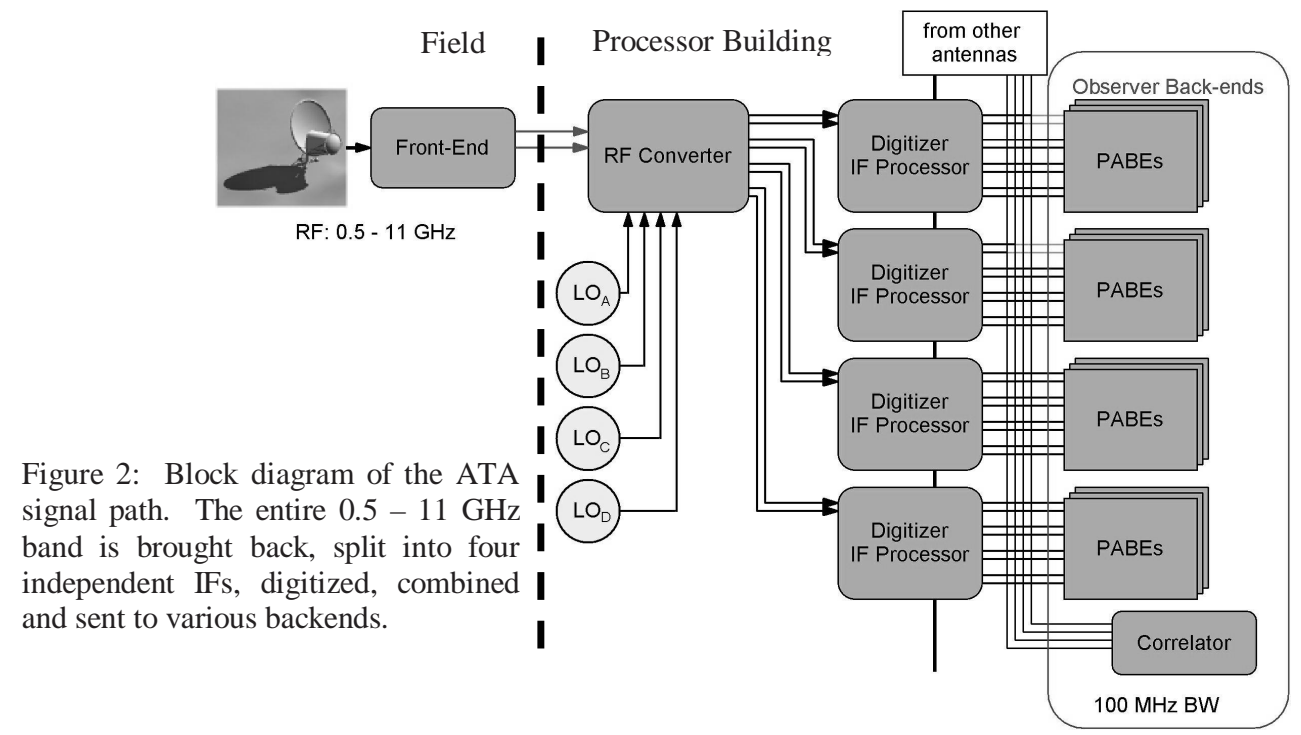

The antenna is an offset Gregorian design, which allows a larger secondary for low frequency performance and also provides greater efficiency in general. The hydroforming technology used to make these surfaces is the same technique used to generate low-cost satellite reflectors by Andersen Manufacturing (Idaho Fall, ID). The structure is rimsupported with minimal back-up structure and sits atop a 18" pipe (Figure 3). The antennas are grouped into zones, where each zone has a "node" from which power, fiber and air radiate to each antenna.

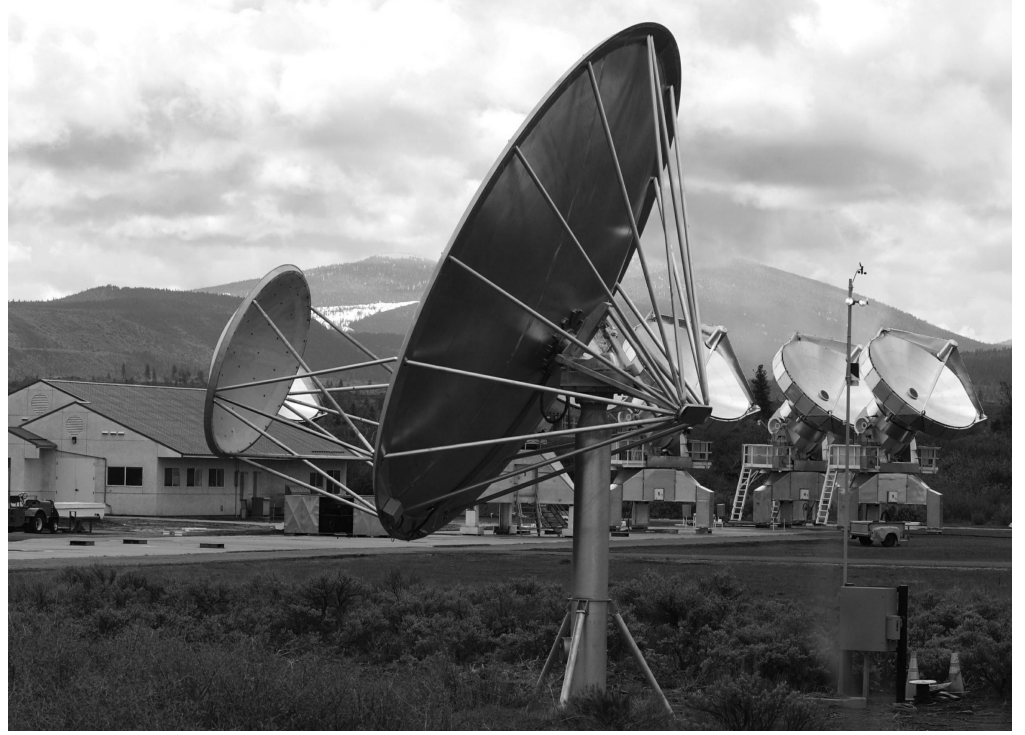

Figure 3: ATA antenna with existing BIMA dishes in background. BIMA is combining with the Caltech Millimeter-Wavelength Array and relocating to the Owen's Valley, CA area (Credit Seth Shostak).

The ATA feed is a pyramidal log-periodic feed (Figure 4) [2]. The dual-linear polarization feed achieves about $12 \mathrm{dBi}$ forward gain and is designed for an equivalent focal-length to antenna diameter ratio (f/D) of 0.65 . This patented feed incorporates a central metallic "pyramid" that allows low-noise amplifiers to be housed in a small cryogenic dewar placed directly behind the antenna terminals to yield a low receiver temperature. In transmission, the feed excites a waveguide mode at the terminals, which travels in the interior space between the arms and pyramid until it meets a resonant condition (at about $\lambda / 2$ ). It is then radiated in free space back towards the small end. The linear dimensions of the feed yield an operating range from about $500 \mathrm{MHz}$ to $15 \mathrm{GHz}$ and a feed focus drive allows any frequency to be 
focused. Focusing to about $6.25 \mathrm{GHz}$ allows the entire band to be accessed with about 1-dB degradation at the band edges.

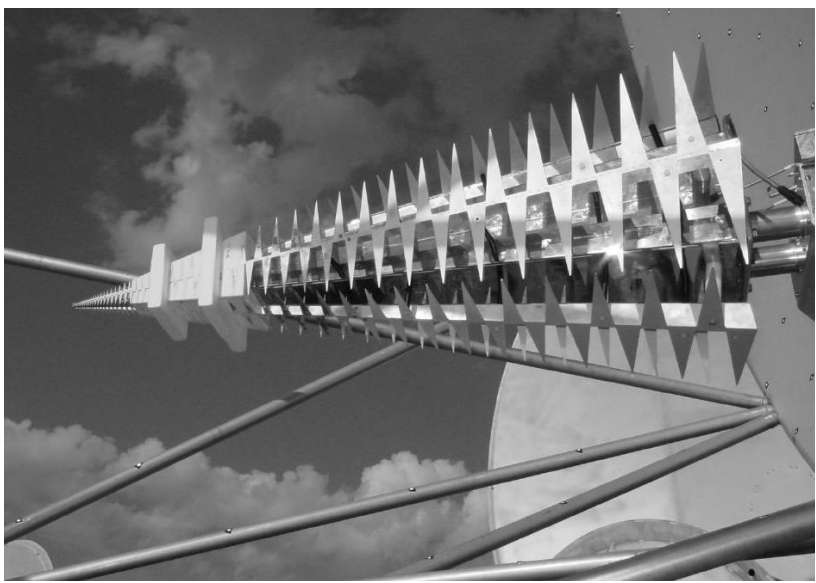

Figure 4: ATA feed on an antenna. Two other antennas are partly visible in the background. (Credit David DeBoer)

The feed geometry requires a pyramidal-shaped dewar with a long, narrow twin-lead to connect the feed terminals to the end of a balun and input/output on opposite ends. To accommodate this geometry, a tapered-line balun was developed to match the balanced $240 \Omega$ antenna terminals to the single-ended low-noise amplifier [3], which is shown in Figure 5. The balun and amplifier reside in the dewar, which will be cooled to about $70 \mathrm{~K}$. Current feeds incorporate a pulse-tube refrigerator designed for the project by NIST and a compressor designed in-house [4].

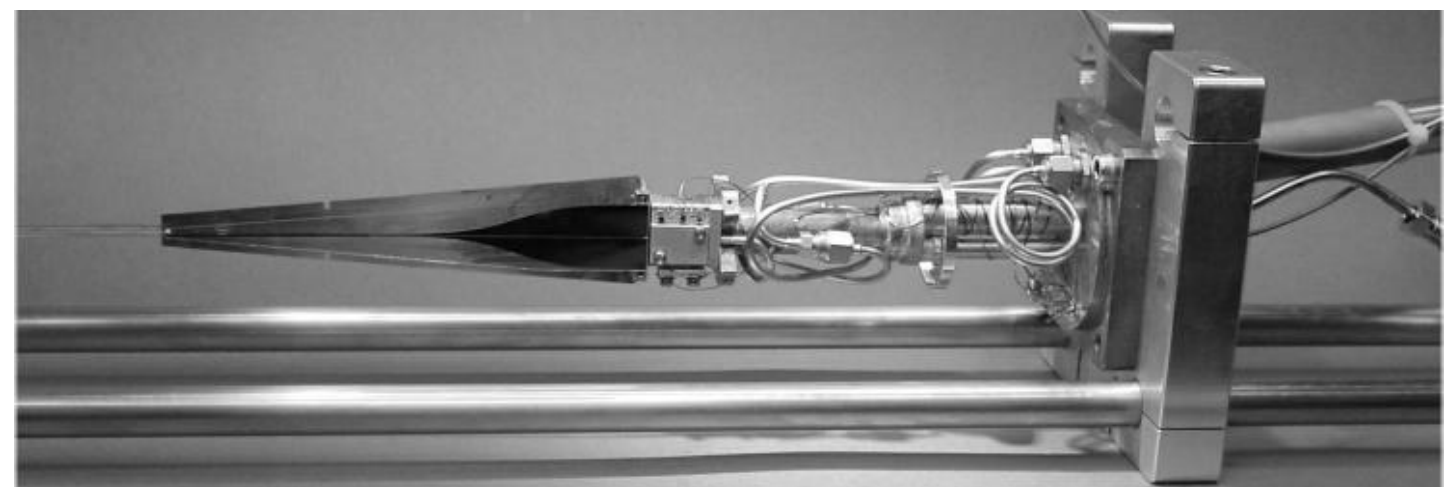

Figure 5: Interior of dewar. The balun, lna and output coax of one polarization is visible. The assembly slides into the dewar housing. (Credit Matt Fleming).

The low-noise amplifier (LNA) is a $0.1 \mu \mathrm{m}$ Indium Phosphide monolithic microwave integrated circuit (MMIC) designed and packaged by Caltech [5,6]. The amplifier contributes approximately $10 \mathrm{~K}$ across the entire band, just under one-quarter of the predicted $45 \mathrm{~K}$ total system temperature when cooled to $77 \mathrm{~K}$. The amplifier feeds a variable gain post-amplifier module utilizing wide-bandwidth RF Micro Devices gain blocks and Hittite variable attenuators. The entire bandwidth $(0.5-11.2 \mathrm{GHz})$ is brought back to a centrally located processing facility via analog fiber-optic links developed for this project by Photonic Systems Inc (Burlington, MA) [7]. The post-amplifiers and photonic links sit in a thermally controlled enclosure at the back of the feed.

The remainder of the signal path will lie within a shielded room within the processor building, which is near the center of the eventual 350-element array (see Figure 6). The RF converter board accepts the two linear polarization fibers from an antenna and passes four independent dual-polarization IF channels (400 MHz each at $1.56 \mathrm{GHz}$ ) to the digitizer, which produces I\&Q digital datastreams at $155 \mathrm{Mbps}$. These streams are transported via fiber to the IF Processor (IFP). The IFP then combines the streams to produce four independent dual-polarization beams per tuning (for a total of 32 beams) at a data rate of about $103 \mathrm{Mbps}$ which are then routed to back-end processors. In addition, all data for two tunings are fed to the correlator at $103 \mathrm{Mbps}$. The digital hardware is based primarily on fieldprogrammable gate arrays (FPGAs). Control and communications of the entire array leverages the ubiquity and performance of the in-built TCP/IP and Java capabilities of microcontrollers. One might call this an application of the 
oft-invoked "Internet-enabled soda pop machine". Initial back-ends will comprise SETI detectors, correlator, and general purpose astronomical spectrometer.

\section{Status}

The foundations and the trenching for the 33 dishes to be emplaced by this fall are installed, as is the man-hole where all of the conduits for 350 antennas will terminate. Some of this is shown in Figures 6 and 7 . Most of the parts for the antenna are either on-hand or on order and assembly will begin Summer of 2004.

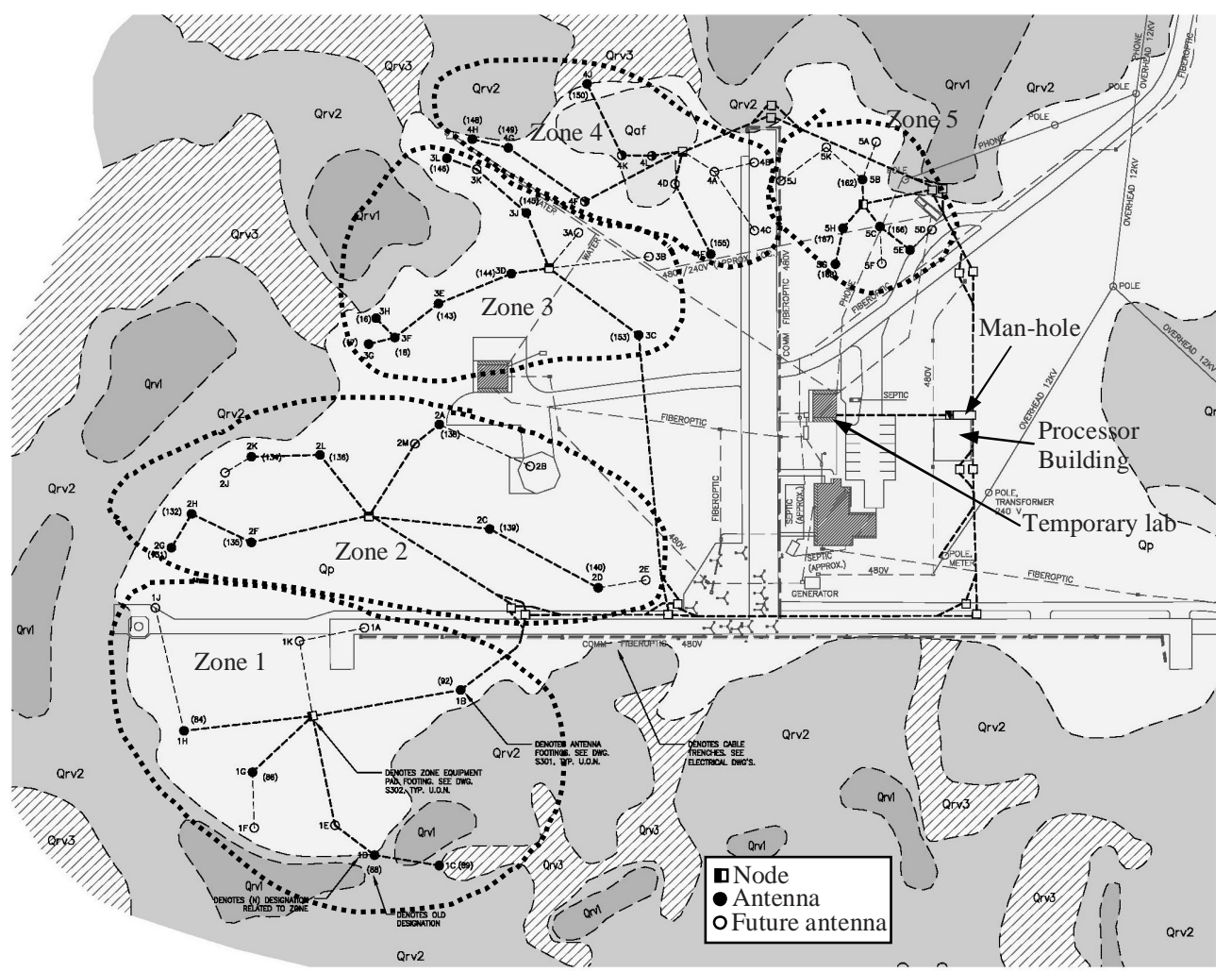

Figure 6: Drawing for emplacement of the next 30 antennas, for a total of 33 by Fall 2004. The zones are marked by the dashed lines. The background is the geological structure, dominated by lava flows.
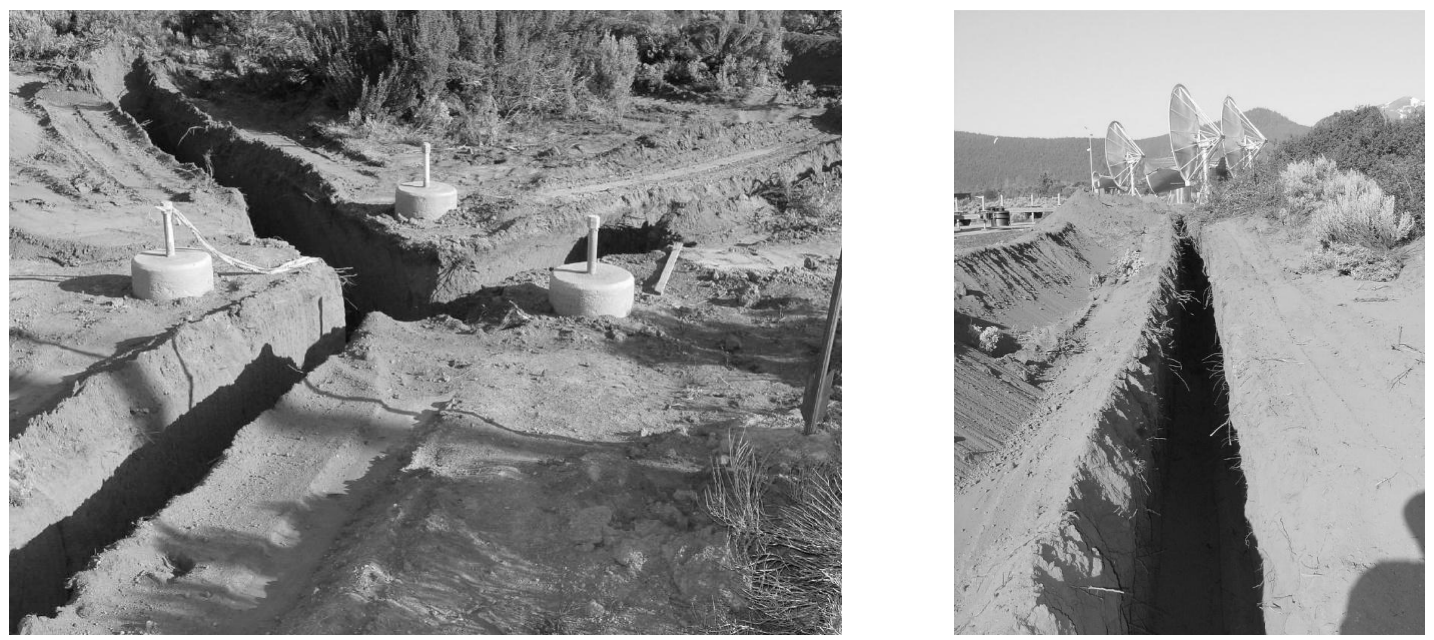

Figure 7: Picture of the trenching threading an antenna foundation (left) and the trench headed towards the three existing ATA dishes. (Credit David DeBoer) 
Prototype feeds are currently being tested on the antennas and prototypes for all signal path elements are under test in the laboratory. Testing of the existing three antennas is on-going, utilizing the signal and control paths from the antenna to the optical fiber receiver in the temporary processing lab. A software correlator, spectrum analyzer and power meter are all being used to characterize the mechanical and electromagnetic performance.

\section{ACKNOWLEDGEMENTS}

The authors wish to acknowledge the support of the Paul G. Allen Charitable Foundations.

\section{REFERENCES}

[1] W.J. Welch and J.W. Dreher, “The One Hectare Telescope,” Proc. SPIE Vol. 4015, p. 8-18, (2000)

[2] G. Engargiola and W.J. Welch, “Log-periodic antenna,” US Patent 6,677,913 (2004)

[3] G. Engargiola, "Tapered microstrip balun for integrating a low noise amplifier with a nonplanar log periodic antenna," Review of Scientific Instruments, Vol 74 Number 12, (2004)

[4] J. B. Lugten, "Low-Cost Cryocoolers from the Allen Telescope Array," Advances in Cryogenic Engineering, vol. $49(2003)$

[5] S. Weinreb, T. Gaier, J. E. Fernandez, N. Erickson, and J. Wielgus, "Cryogenic Low Noise Amplifiers", GaAs2000 Symposium, Paris, October 2, 2000.

[6] S. Weinreb and N. Wadefalk, private communication (2003)

[7] E. Ackerman, C. Cox, J. Dreher, M. Davis, and D. DeBoer, "Fiber-Optic Antenna Remoting for Radioastronomy Applications," URSI $27^{\text {th }}$ General Assembly, Maastricht.

\section{Appendix A. ATA Signal Path}

As a joint project intended for large surveys, commensal-use of the array is paramount. This is enabled by the large field-of-view, wide bandwidth brought back to the lab, and flexible digital hardware. This allows the SETI Institute to conduct a full-time, full-sensitivity targeted search for extraterrestrial technological signals concurrently with Radio Astronomy Lab-conducted wide surveys of $\mathrm{HI}$ using the correlator. In addition, other back-ends (e.g. the spectrometer) can utilize other available beams. The "scarce" resources will be the pointing direction of the antennas (of which there is one, unless sub-arrays are used) and the tunings (of which there are four). Figure 8 shows a more detailed block diagram of the signal path as it relates to the phased-array back-ends, indicating how it is structured by "card". The correlator gets the data-streams from all the antennas for two tunings, so that it can form images of the entire field-ofview at two independent frequencies (this constrains four of the 32 beams, which are still available but point at boresight - denoted by the open arrows in Figure 8).

Figure 9 indicates the multiple-use capability (although it is shown at only one frequency instead of four). The large circle is the 3-dB field-of-view of the ATA at $1.2 \mathrm{GHz}$ (contrasted with the Arecibo field-of-view) and the 32 smaller circles are the independent ATA beams (contrasted with the Arecibo resolution, which is the same as the field-of-view). These beams can point anywhere in the sky, however they will typically point within the main beam of the primary. The cross-hatch is to demonstrate that the correlator will also image the entire field-of-view.

Figure 10 shows the up/down conversion scheme to allow the flexible tuning ability. Four tunable LO's (tuning between about 16.3 and $26.6 \mathrm{GHz}$ ) and two fixed LO's bring the signals down to baseband, where they are digitized in quadrature at about 155 Mbps. The IFP time delays, down-samples to about $103 \mathrm{Mbps}$, provides a fine phase correction, sums and passes the data on. In addition, it provides the individual antenna data streams for two tunings to the correlator. The final 32 sums are passed on to the back-ends. 


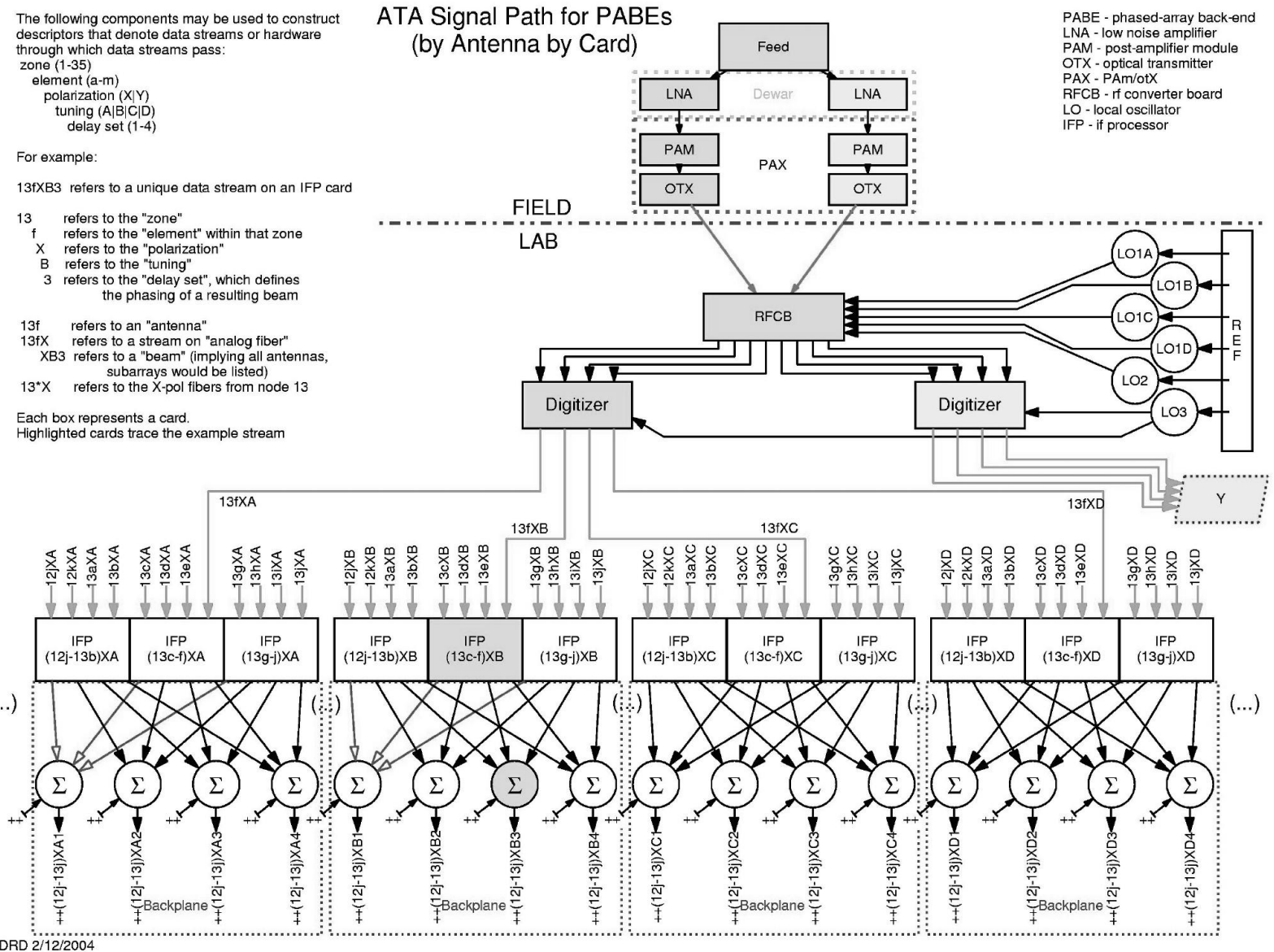

Figure 8: Detailed block diagram of the ATA as it relates to the phased-array back-ends (i.e., not the correlator), indicating structure by "card". It also shows the naming convention of the data streams.

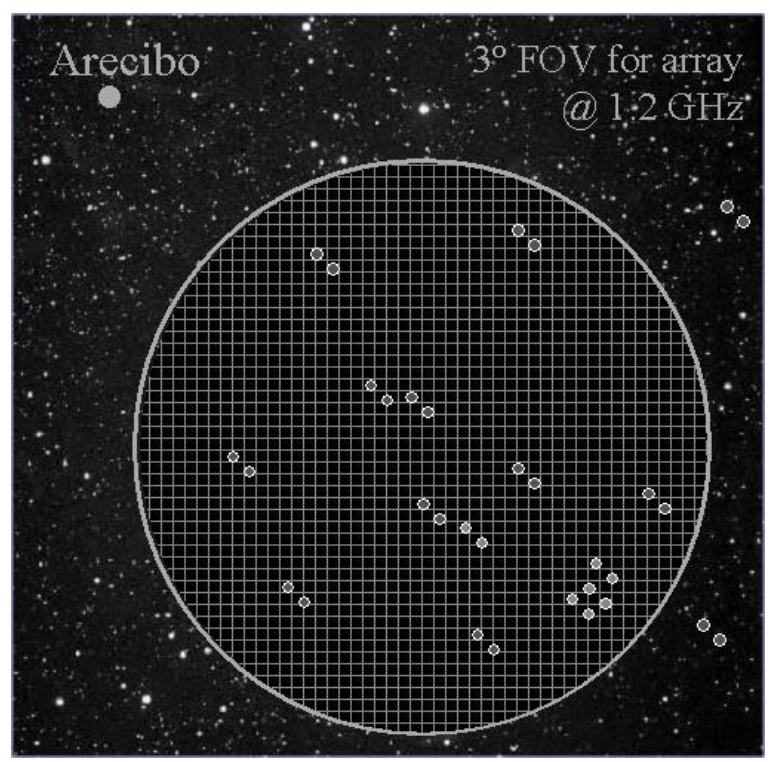

Figure 9: Flexibility of the ATA. The large field-of-view and the distributed array allow effective multiple concurrent-use of the array. 


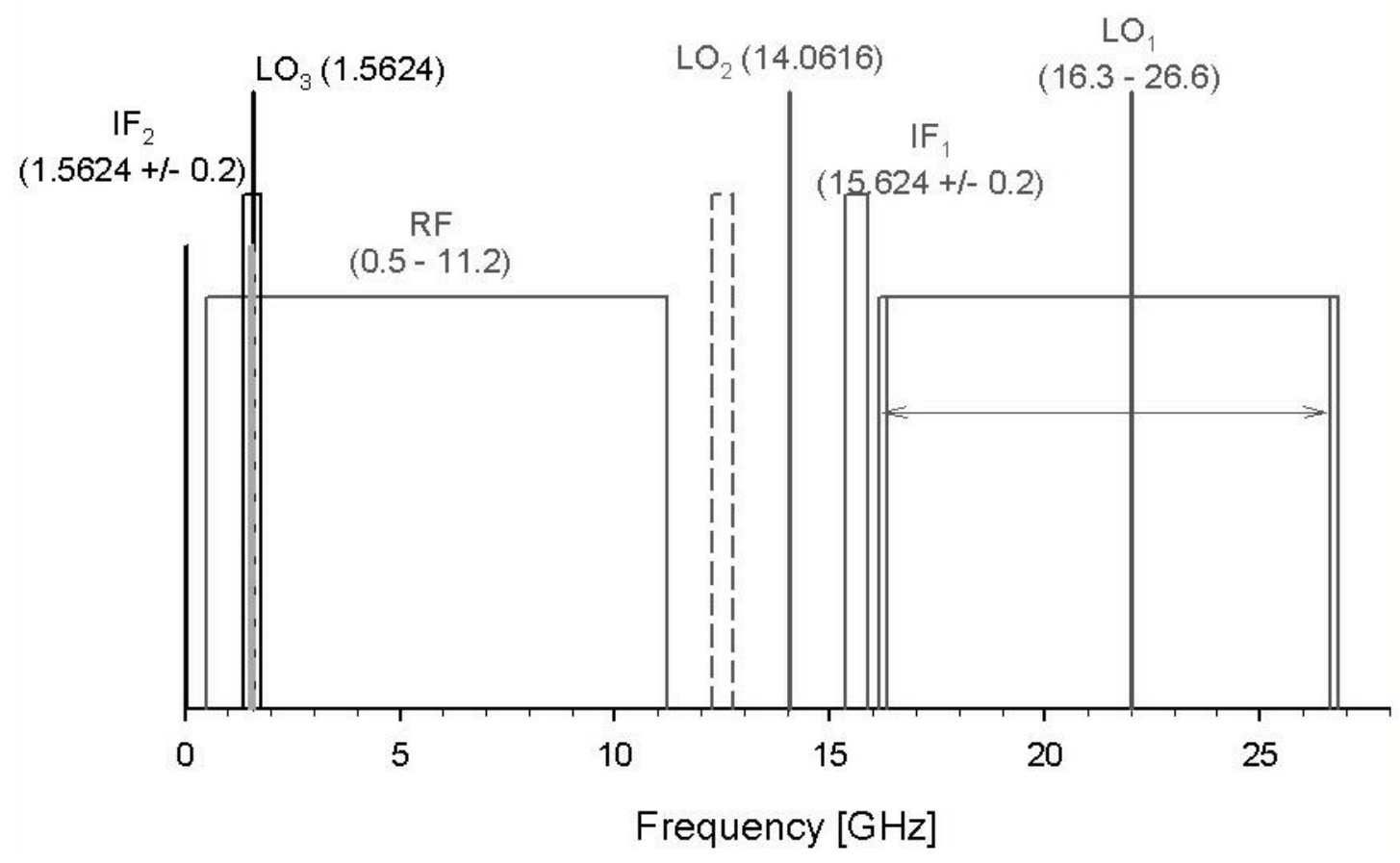

Figure 10: Up/down conversion scheme for the RF converter board. The IF2 output from this board feeds the digitizer, which converts down to baseband and samples. 